Economics Department

Central Limit Theorem for Asymmetric Kernel Functionals

Marcelo Fernandes

ECO No. 2000/1

EUI WORKING PAPERS

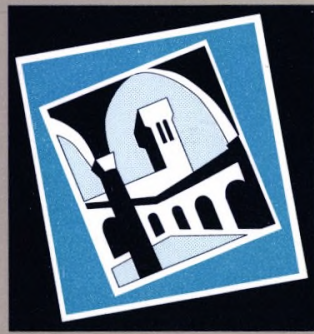

EUROPEAN UNIVERSITY INSTITUTE 
European University Institute

30001003332568 
EUROPEAN UNIVERSITY INSTITUTE, FLORENCE

ECONOMICS DEPARTMENT

EUI Working Paper ECO No. 2000/1

WP 330

EUR

Central Limit Theorem

for Asymmetric Kernel Functionals

MARCELO FERNANDES

BADIA FIESOLANA, SAN DOMENICO (FI) 
All rights reserved.

No part of this paper may be reproduced in any form without permission of the author.

(C) 2000 Marcelo Fernandes

Printed in Italy in March 2000

European University Institute

Badia Fiesolana

I - 50016 San Domenico (FI)

Italy 


\title{
Central Limit Theorem for Asymmetric Kernel Functionals
}

\author{
Marcelo Fernandes
}

February 2000

\begin{abstract}
Asymmetric kernels are quite useful for the estimation of density functions which have bounded support. Gamma kernels are designed to handle density functions whose supports are bounded from one end only, whereas beta kernels are particularly convenient for the estimation of density functions with compact support. These asymmetric kernels are non-negative and free of boundary bias. Moreover, their shape varies according to the location of the data point, thus also changing the amount of smoothing. This paper extends the central limit theorem for degenerate U-statistics in order to compute the limiting distribution of certain asymmetric kernel functionals.
\end{abstract}




\section{Introduction}

Fixed kernels are not appropriate to estimate density functions whose supports are bounded in view that they engender boundary bias due to the allocation of weight outside the support in the event that smoothing is applied near the boundary. A proper asymmetric kernel never assigns weight outside the density support and therefore should produce better estimates of the density near the boundary. Indeed, Chen (1999a,b) showed that replacing fixed kernels with asymmetric kernels increases substantially the precision of density estimation close to the boundary. In particular, beta kernels are particularly appropriate to estimate densities with compact support, whereas gamma kernels are more convenient to handle density functions whose supports are bounded from one end only. These asymmetric kernels are non-negative and free of boundary bias. Moreover, their shape varies according to the location of the data point, thus also changing the amount of smoothing.

The aim of this paper is to build on Hall's (1984) central limit theorem for degenerate U-statistics in order to derive asymptotics for asymmetric kernel functionals. The motivation is simple. It is often the case that one must derive the limiting distribution of density functionals such as

$I=\int_{A} \varphi(x) \hat{f}^{2}(x) \mathrm{d} x$

where the support $A$ is bounded. Examples abound in econometrics and statistics. Indeed, a central limit theorem for the density functional (1) is useful to study the order of closeness between the integrated square error and the mean integrated squared error in the ambit of non-parametric kernel density estimation. Although there are sharp results for nonparametric density estimation based on fixed kernels (Bickel and Rosenblatt, 1973; Hall, 1984), no results are available for asymmetric kernel density estimation.

Furthermore, goodness-of-fit test statistics are usually driven by second-order asymptotics (e.g. Aït-Sahalia, 1996 and Aït-Sahalia, Bickel 
and Stoker, 1998), so that density functionals such as (1) arise very naturally in that context. Consider, for instance, one of the goodness-offit tests advanced by Fernandes and Grammig (1999) for duration models gauges how large is

$$
\Lambda(f, \theta)=\int_{0}^{\infty} w(x)\left[\Gamma_{\theta}(x)-\Gamma_{f}(x)\right]^{2} f(x) \mathrm{d} x,
$$

where $w(x)$ is a trimming function and $\Gamma_{f}(\cdot)$ and $\Gamma_{\theta}(\cdot)$ denote the nonand parametric hazard rate functions, respectively. It follows from the functional delta method that the asymptotic behaviour of $(2)$ is driven by the leading term of the second functional derivative, namely

$$
\int_{0}^{\infty} \varphi(x)[\hat{f}(x)-f(x)]^{2} \mathrm{~d} x=2 \int_{0}^{\infty} w(x) \frac{\Gamma_{f}(x)}{1-F(x)}[\hat{f}(x)-f(x)]^{2} \mathrm{~d} x .
$$

Note that duration data are non-negative by definition, hence it is convenient to utilise gamma kernels to avoid boundary bias in the density estimation.

The remainder of this paper is organised as follows. In section 2, I review the properties of beta and gamma kernels. In sections 3 and 4, I apply Hall's (1984) central limit theorem for degenerate U-statistics to derive the limiting distribution of gamma and beta kernel functionals, respectively.

\section{Asymmetric kernels}

Let $X_{1}, \ldots, X_{T}$ be a random sample from an unknown probability density function $f$ defined on a bounded support $A$. In what follows, I consider that $A$ is either bounded from one end or compact. Without loss of generality, I assume that $A=[0, \infty)$ in the first case, whereas $A=[0,1]$ in the second context. Finally, assume that the density function $f$ and its second order derivative are bounded and uniformly continuous on the real line. 
Instead of the usual non-parametric kernel density estimator

$\hat{f}(x)=\frac{1}{T h} \sum_{t=1}^{T} K\left(\frac{x-X_{t}}{h}\right)$,

where $K$ is a fixed kernel function and $h$ is a smoothing bandwidth, consider the asymmetric kernel estimator

$\hat{f}(x)=\frac{1}{T b} \sum_{t=1}^{T} K_{A}\left(X_{t}\right)$

where $K_{A}(\cdot)$ corresponds either to the gamma kernel

$K_{x / b+1, b}(u)=\frac{u^{x / b} \exp (-u / b)}{\Gamma(x / b+1) b^{x / b}} I\{u \in[0, \infty)\}$

or to the beta kernel

$K_{x / b+1,(1-x) / b+1}(u)=\frac{u^{x / b}(1-u)^{(1-x) / b}}{B(x / b+1,(1-x) / b+1)} I\{u \in[0,1]\}$

according to the support under consideration.

Chen (1999a,b) showed that both estimators are boundary bias free in view that the bias is of order $O(b)$ both near the boundaries and in the interior of the support. The absence of boundary bias is due to the fact that asymmetric kernels have the same support of the underlying density, and hence no weight is assigned outside the density support. The trick is that asymmetric kernel functions are flexible enough to vary their shape (and thus the amount of smoothing) according to the location of $x$ within the support.

On the other hand, the asymptotic variance of asymmetric kernels is of higher order $O\left(T^{-1} b^{-1}\right)$ near the boundaries than in the interior, which is of order $O\left(T^{-1} b^{-1 / 2}\right)$. Nonetheless, this has negligible impact on the integrated variance, thus it does affect the mean integrated square error. Furthermore, it is possible to show that the optimal bandwidth $b_{*}=$ $O\left(T^{-2 / 5}\right)=O\left(h_{*}^{2}\right)$, where $h_{*}$ is the optimal bandwidth for fixed kernel 
estimators. Accordingly, both beta and gamma kernel density estimators achieve the optimal rate of convergence for the mean integrated squared error of non-negative kernels. ${ }^{1}$ Lastly, a unique feature for the gamma kernel estimator is that its variance decreases as $x$ increases, though at the expense of an upping in the bias.

\section{Gamma kernel functionals}

The asymptotic behaviour of gamma kernel functionals of the form (1) is derived using U-statistic theory. For this reason, I utilise a decomposition which forces a degenerate U-statistic to emerge. Let $r_{T}(x, X)=$ $\varphi^{1 / 2}(x) K_{x / b+1, b}\left(X_{t}\right), \breve{r}_{T}(x, X)=r_{T}(x, X)-E_{X}\left[r_{T}(x, X)\right]$ and $\int_{u}$ denote the integral over the support of $u$. Then,

$$
\begin{aligned}
I & =\int_{x}\left[\sum_{t=1}^{T} r_{T}\left(x, X_{t}\right) / T\right]^{2} \mathrm{~d} x \\
& =\frac{1}{T^{2}} \sum_{s, t} \int_{x} r_{T}\left(x, X_{t}\right) r_{T}\left(x, X_{s}\right) \mathrm{d} x \\
& =I_{1}+I_{2}+I_{3}+I_{4},
\end{aligned}
$$

where

$$
\begin{aligned}
I_{1} & =\frac{2}{T^{2}} \sum_{s<t} \int_{x} \breve{r}_{T}\left(x, X_{t}\right) \breve{r}_{T}\left(x, X_{s}\right) \mathrm{d} x \\
I_{2} & =\frac{1}{T^{2}} \sum_{t} \int_{x} r_{T}^{2}\left(x, X_{t}\right) \mathrm{d} x \\
I_{3} & =\frac{T(T-1)}{T^{2}} \int_{x} E_{X}^{2}\left[r_{T}(x, X)\right] \mathrm{d} x \\
I_{4} & =\frac{2 T(T-1)}{T^{2}} \int_{x} \breve{r}_{T}\left(x, X_{t}\right) E_{X}\left[r_{T}(x, X)\right] \mathrm{d} x .
\end{aligned}
$$

The first term stands for a degenerate U-statistic and will contribute with the variance in the limiting distribution. The second term will

1 Non-negative kernels define the class of second order kernel functions. Higher order kernels may bring about some bias reduction at the expense of assuming negative values (see Müller, 1984, for a list). 
contribute with the asymptotic mean, hence it may be interpreted as some sort of asymptotic bias of the functional estimator depending on the context. The third and the fourth terms are, in turn, negligible under a proper choice of the bandwidth $b$. Suppose the bandwidth $b$ is such that $T b^{9 / 4}$ shrinks to zero as sample size $T$ grows. This assumption implies some degree of undersmoothing in view that Chen (1999b) has shown that the optimal bandwidth for gamma kernels is of order $O\left(T^{-2 / 5}\right)$.

I start by deriving the first two moments of $r_{T}(x, X)$. Note that

$E_{X}\left[r_{T}(x, X)\right]=\varphi^{1 / 2}(x) \int_{X} K_{x / b+1, b}(X) f(X) \mathrm{d} X=\varphi^{1 / 2}(x) E_{\zeta}[f(\zeta)]$,

where $\zeta \sim \mathcal{G}(x / b+1, b)$. The mean and variance of a $\mathcal{G}(\mu, v)$ are simply $\mu v$ and $\mu v^{2}$, respectively. Therefore, applying a Taylor expansion yields

$$
\begin{aligned}
E_{\zeta}[f(\zeta)] & =f\left(E_{\zeta}\right)+\frac{1}{2} f^{\prime \prime}(x) V_{\zeta}+o(b) \\
& =f(x+b)+\frac{1}{2} f^{\prime \prime}(x)(x+b) b+o(b) \\
& =f(x)+b\left[f^{\prime}(x)+\frac{1}{2} f^{\prime \prime}(x)\right]+o(b) .
\end{aligned}
$$

It is noteworthy that the last expression demonstrates that the gamma kernel estimation of the density function has a uniform bias of order $O(b)$. Put differently, the order of magnitude of the bias does not depend on the position of $x$, that is, whether it is close to the origin or in the interior of the support. To sum up, $E_{X}\left[r_{T}(x, X)\right]=\varphi^{1 / 2}(x) f(x)+O(b)$, which implies that $\breve{r}_{T}(x, X)=O(b)$.

The second moment of $r_{T}(x, X)$ is computed in similar way. It follows from Chen's (1999b) derivation of the variance of the gamma kernel estimator that

$$
\begin{aligned}
E_{X}\left[r_{T}^{2}(x, X)\right] & =\varphi(x) \int_{X} K_{x / b+1, b}^{2}(X) f(X) \mathrm{d} X \\
& =\varphi(x) B_{b}(x) E_{\eta}[f(\eta)],
\end{aligned}
$$

where

$$
B_{b}(x)=\frac{\Gamma(2 x / b+1) / b}{2^{2 x / b+1} \Gamma^{2}(x / b+1)}
$$


and $\eta \sim \mathcal{G}(2 x / b+1, b)$. Hence applying a Taylor expansion yields

$$
\begin{aligned}
E_{\eta}[f(\eta)] & =f\left(E_{\eta}\right)+\frac{1}{2} f^{\prime \prime}(x) V_{\eta}+o(b) \\
& =f(2 x+b)+\frac{1}{2} f^{\prime \prime}(x)(2 x+b) b+o(b) \\
& =f(x)+f^{\prime}(x) x+b\left[f^{\prime}(x)+f^{\prime \prime}(x) x\right]+o(b) \\
& =f(x)+f^{\prime}(x) x+O(b) .
\end{aligned}
$$

It follows then that

$$
\begin{aligned}
E\left(I_{2}\right) & =\frac{1}{T} \int_{x} E_{X}\left[r_{T}^{2}(x, X)\right] \mathrm{d} x \\
& =\frac{1}{T} \int_{x} \varphi(x) B_{b}(x)\left[f(x)+f^{\prime}(x) x+O(b)\right] \mathrm{d} x \\
& =\frac{1}{T} \int_{x} \varphi(x) B_{b}(x) f(x) \mathrm{d} x+O(1 / T)
\end{aligned}
$$

For $b$ small enough, Chen (1999b) approximates $B_{b}(x)$ according to the behaviour of $x / b$. The motivation stems from the fact that, in the interior of the support, $x / b$ grows without bound as $b$ shrinks to zero, whereas $x / b$ converges to some non-negative constant $c$ in the boundary. The decomposition dictates that

$B_{b}(x) \sim\left\{\begin{array}{cc}\frac{1}{2 \sqrt{\pi}} b^{-1 / 2} x^{-1 / 2} & \text { if } x / b \rightarrow \infty \\ \frac{\Gamma(2 c+1) / b}{2^{2 c+1} \Gamma^{2}(c+1)} & \text { if } x / b \rightarrow c,\end{array}\right.$

which implies that $B_{b}(x)$ is higher near the origin. Nonetheless, I show that there is no impact whatsoever in $E\left(I_{2}\right){ }^{2}$

Let $\delta=b^{1-\epsilon}$, where $0<\epsilon<1$. Then,

$$
E\left(I_{2}\right)=\frac{1}{T} \int_{x} \varphi(x) B_{b}(x) f(x) \mathrm{d} x+O(1 / T)
$$

2 This result is analogous to Chen's (1999b) result concerning the variance of the gamma kernel estimator. In particular, the variance mounts as $x$ approaches the boundary, but this increase does not affect the integrated variance of the estimator. 


$$
\begin{aligned}
& =\frac{1}{T} \int_{0}^{\delta}+\int_{\delta}^{\infty} \varphi(x) B_{b}(x) f(x) \mathrm{d} x+O(1 / T) \\
& =\frac{1}{2 \sqrt{\pi} T} \int_{\delta}^{\infty} b^{-1 / 2} x^{-1 / 2} \varphi(x) f(x) \mathrm{d} x+O\left(T^{-1} b^{-\epsilon}\right) \\
& =\frac{b^{-1 / 2}}{2 \sqrt{\pi} T} \int_{x} \varphi(x) x^{-1 / 2} f(x) \mathrm{d} x+o\left(T^{-1} b^{-1 / 2}\right)
\end{aligned}
$$

provided that $\epsilon$ is properly chosen and $E\left[\varphi(x) x^{-1 / 2}\right]$ is finite. Therefore, it ensues that

$T b^{1 / 4} E\left(I_{2}\right)=\frac{b^{-1 / 4}}{2 \sqrt{\pi}} E\left[x^{-1 / 2} \varphi(x)\right]$.

Notice also that

$$
\begin{aligned}
V\left(I_{2}\right) & =\frac{1}{T^{3}} E\left[\int_{x} r_{T}^{2}(x, X) \mathrm{d} x\right]^{2}-\frac{1}{T^{3}} E^{2}\left[\int_{x} r_{T}^{2}(x, X) \mathrm{d} x\right] \\
& =\frac{1}{T^{3}} E\left[\int_{x} r_{T}^{2}(x, X) \mathrm{d} x\right]^{2}-\frac{1}{T^{3}}\left[\int_{x} E r_{T}^{2}(x, X) \mathrm{d} x\right]^{2} \\
& =O\left(T^{-3} b^{-1}\right) .
\end{aligned}
$$

Thus, $V\left(T b^{1 / 4} I_{2}\right)=T^{2} b^{1 / 2} V\left(I_{2}\right)=O\left(T^{-1} b^{-1 / 2}\right)$, which is of order $o(1)$ given the assumption on the bandwidth. Thus, by Chebyshev's inequality,

$$
T b^{1 / 4} I_{2}-\frac{b^{-1 / 4}}{2 \sqrt{\pi}} E\left[x^{-1 / 2} \varphi(x)\right]=o_{p}(1) .
$$

The fact that $b=o\left(T^{-4 / 9}\right)$ also ensures that the third and fourth terms are negligible if properly normalised. Indeed, it follows from

$I_{3}=\frac{T-1}{T} \int_{x} E^{2}\left[r_{T}(x, X)\right] \mathrm{d} x=\frac{T-1}{T} O\left(b^{2}\right)=O\left(b^{2}\right)$

that $T b^{1 / 4} I_{3}=O\left(T b^{9 / 4}\right)$, which is $o(1)$ by assumption. Furthermore, $I_{4}=\frac{2(T-1)}{T^{2}} \sum_{t=1}^{T} \int_{x} \breve{r}_{T}\left(x, X_{t}\right) E_{X} r_{T}(x, X) \mathrm{d} x$ 
and hence

$E\left(I_{4}\right)=\frac{2(T-1)}{T} \int_{x} E_{X}\left[\breve{r}_{T}(x, X)\right] E_{X}\left[r_{T}(x, X)\right] \mathrm{d} x=0$

given that $\check{r}_{T}(x, X)$ has zero mean. Besides,

$E_{X}\left\{\int_{x} \breve{r}_{T}\left(x, X_{t}\right) E_{X}\left[r_{T}(x, X)\right] \mathrm{d} x\right\}^{2}=O\left(b^{2}\right)$,

which implies that $E\left(I_{4}^{2}\right)=O\left(T^{-1} b^{2}\right)$ and therefore

$E\left(T b^{1 / 4} I_{4}\right)^{2}=T^{2} b^{1 / 2} E\left(I_{4}^{2}\right)=O\left(T b^{5 / 2}\right)=o(1)$.

Afresh, it stems from Chebyshev's inequality that $T b^{1 / 4} I_{4}=o_{p}(1)$.

Finally, recall that $I_{1}=\sum_{s<t} H_{T}\left(X_{t}, X_{s}\right)$, where

$H_{T}\left(X_{t}, X_{s}\right)=\frac{2}{T^{2}} \int_{x} \breve{r}_{T}\left(x, X_{t}\right) \breve{r}_{T}\left(x, X_{s}\right) \mathrm{d} x$.

Then, $I_{1}$ is a degenerate U-statistic in view that $H_{T}\left(X_{t}, X_{s}\right)$ is symmetric, centred, and $E\left[H_{T}\left(X_{t}, X_{s}\right) \mid X_{s}\right]=0$ almost surely. To see why, note that

$$
\begin{aligned}
E\left[H_{T}\left(X_{t}, X_{s}\right) \mid X_{s}\right] & =\frac{2}{T^{2}} \int_{x} \breve{r}_{T}\left(x, X_{s}\right) E\left[\breve{r}_{T}\left(x, X_{t}\right) \mid X_{s}\right] \mathrm{d} x \\
& =\frac{2}{T^{2}} \int_{x} \breve{r}_{T}\left(x, X_{s}\right) E\left[\breve{r}_{T}\left(x, X_{t}\right)\right] \mathrm{d} x
\end{aligned}
$$

in view of the independence between $X_{t}$ and $X_{s}$. It suffices then to observe that $\breve{r}_{T}\left(x, X_{t}\right)$ has by construction zero mean. Thereby, I apply Hall's (1984) central limit theorem for degenerate U-statistics, which states that if

$$
\frac{E_{X_{1}, X_{2}}\left\{E_{X_{1}}^{2}\left[H_{T}\left(X_{1}, X_{1}\right) H_{T}\left(X_{1}, X_{2}\right)\right]\right\}+\frac{1}{T} E_{X_{1}, X_{2}}\left[H_{T}^{4}\left(X_{1}, X_{2}\right)\right]}{E_{X_{1}, X_{2}}^{2}\left[H_{T}^{2}\left(X_{1}, X_{2}\right)\right]} \rightarrow 0
$$

as sample size grows, then

$$
I_{1} \stackrel{d}{\longrightarrow} N\left(0, \frac{T^{2}}{2} E_{X_{1}, X_{2}}\left[H_{T}^{2}\left(X_{1}, X_{2}\right)\right]\right) .
$$


Tedious algebra shows that (6) holds. Indeed, the two terms of the numerator are of order $O\left(T^{-12} b^{-2}\right)$ and $O\left(T^{-9} b^{-3 / 2}\right)$, respectively, whereas the denominator is of order $O\left(T^{-8} b^{-1}\right)$. In what follows, I demonstrate the last assertion as a by-product of the derivation of the asymptotic variance above.

Let $V_{H}=\frac{T^{4}}{2} E_{X_{1}, X_{2}}\left[H_{T}^{2}\left(X_{1}, X_{2}\right)\right]$, then

$$
\begin{aligned}
V_{H}= & 2 \int_{X_{1}, X_{2}}\left[\int_{x} \breve{r}_{T}\left(x, X_{1}\right) \check{r}_{T}\left(x, X_{2}\right) \mathrm{d} x\right]^{2} f\left(X_{1}, X_{2}\right) \mathrm{d}\left(X_{1}, X_{2}\right) \\
= & 2 \int_{x, y}\left[\int_{X} \breve{r}_{T}(x, X) \breve{r}_{T}(y, X) f(X) \mathrm{d} X\right]^{2} \mathrm{~d}(x, y) \\
= & 2 \int_{x, y} \varphi(x) \varphi(y) E_{X}^{2}\left\{\left[K_{x / b+1, b}(X)-E_{K(x, b)}\right]\right. \\
& \left.\times\left[K_{y / b+1, b}(X)-E_{K(y, b)}\right]\right\} \mathrm{d}(x, y),
\end{aligned}
$$

where $E_{K(u, b)}=E_{X}\left[K_{u / b+1, b}(X)\right]$. Then, it ensues that

$V_{H}=2 \int_{x, y} \varphi(x) \varphi(y)\left[\int_{X} K_{x / b+1, b}(X) K_{y / b+1, b}(X) \mathrm{d} F(X)\right]^{2} \mathrm{~d}(x, y)+O\left(b^{2}\right)$

due to the fact that

$$
\begin{aligned}
E_{K(x, b)} E_{K(y, b)} & =\int_{X} K_{x / b+1, b}(X) E_{K(y, b)} \mathrm{d} F(X) \\
& =\int_{X} E_{K(x, b)} K_{y / b+1, b}(X) \mathrm{d} F(X) \\
& =\int_{X} E_{K(x, b)} E_{K(y, b)} \mathrm{d} F(X) \\
& =O\left(b^{2}\right) .
\end{aligned}
$$

Let $g(X)=f(X) K_{x / b+1, b}(X)$, then

$$
V_{H}=2 \int_{x, y} \varphi(x) \varphi(y)\left[\int_{X} g(X) \mathrm{d} K_{y / b+1, b}(X)\right]^{2} \mathrm{~d}(x, y)+O\left(b^{2}\right)
$$


Applying a Taylor expansion gives

$$
\begin{aligned}
\int_{X} g(X) \mathrm{d} K_{y / b+1, b}(X) & =E_{\mathcal{G}(y / b+1, b)}[g(X)] \\
& =g\left[E_{\mathcal{G}(y / b+1, b)}(X)\right]+\frac{g^{\prime \prime}(y) V_{\mathcal{G}(y / b+1, b)}(X)}{2}+o(b) \\
& =g(y+b)+\frac{1}{2} g^{\prime \prime}(y)(y+b) b+o(b) \\
& =g(y)+b\left[g^{\prime}(y)+\frac{1}{2} g^{\prime \prime}(y) y\right]+o(b) . \\
& =g(y)+O(b) .
\end{aligned}
$$

This means that

$$
\begin{aligned}
V_{H} & =2 \int_{x, y} \varphi(x) \varphi(y)\left[f(y) K_{x / b+1, b}(y)\right]^{2} \mathrm{~d}(x, y)+O\left(b^{2}\right) \\
& =2 \int_{x} \varphi(x)\left[\int_{y} \varphi(y) f^{2}(y) K_{x / b+1, b}^{2}(y) \mathrm{d} y\right] \mathrm{d} x+O\left(b^{2}\right) \\
& =2 \int_{x} \varphi(x)\left[\int_{y} h(y) \mathrm{d} K_{x / b+1, b}(y)\right] \mathrm{d} x+O\left(b^{2}\right),
\end{aligned}
$$

where $h(y)=\varphi(y) f^{2}(y) K_{x / b+1, b}(y)$. Afresh, by Taylor expanding, it yields

$$
\begin{aligned}
\int_{y} h(y) \mathrm{d} K_{x / b+1, b}(y) & =E_{\mathcal{G}(x / b+1, b)}[h(y)] \\
& =h\left[E_{\mathcal{G}(x / b+1, b)}(y)\right]+\frac{1}{2} h^{\prime \prime}(x) V_{\mathcal{G}(x / b+1, b)}(y)+o(b) \\
& =h(x+b)+\frac{1}{2} h^{\prime \prime}(x)(x+b) b+o(b) \\
& =h(x)+b\left[h^{\prime}(x)+\frac{1}{2} h^{\prime \prime}(x) x\right]+o(b) \\
& =h(x)+O(b) .
\end{aligned}
$$

Therefore,

$$
\begin{aligned}
V_{H} & =2 \int_{x} \varphi(x) h(x) \mathrm{d} x+O(b) \\
& =2 \int_{x} \varphi^{2}(x) f^{2}(x) K_{x / b+1, b}(x) \mathrm{d} x+O(b) \\
& =2 \int_{x} \varphi^{2}(x) f(x) K_{x / b+1, b}(x) \mathrm{d} F(x)+O(b) .
\end{aligned}
$$


Notice however that using the same technique it is possible to show that $\int_{X} f(X) K_{x / b+1, b}^{2}(X) \mathrm{d} X=K_{x / b+1, b}(x) f(x)+O(b)$.

Hence, it follows that

$$
\begin{aligned}
V_{H} & =2 \int_{x} \varphi^{2}(x) f(x) K_{x / b+1, b}(x) \mathrm{d} F(x)+O(b) \\
& =2 \int_{x} \varphi^{2}(x)\left[\int_{X} f(X) K_{x / b+1, b}^{2}(X) \mathrm{d} X\right] \mathrm{d} F(x)+O(b) \\
& =2 \int_{x} \varphi^{2}(x) B_{b}(x)[f(x)+O(b)] \mathrm{d} F(x)+O(b) \\
& =2 \int_{x} \varphi^{2}(x) B_{b}(x) f(x) \mathrm{d} F(x)+O(b) .
\end{aligned}
$$

By decomposing the integral according to $\delta=b^{1-\epsilon}$, it yields

$$
\begin{aligned}
V_{H} & =\int_{0}^{\delta}+\int_{\delta}^{\infty} 2 \varphi^{2}(x) B_{b}(x) f(x) \mathrm{d} F(x)+O(b) \\
& =\frac{b^{-1 / 2}}{\sqrt{\pi}} \int_{\delta}^{\infty} \varphi^{2}(x) x^{-1 / 2} f(x) \mathrm{d} F(x)+O\left(b^{-\epsilon}\right) \\
& =\frac{b^{-1 / 2}}{\sqrt{\pi}} \int_{x} \varphi^{2}(x) x^{-1 / 2} f(x) \mathrm{d} F(x)+O\left(b^{-1 / 2}\right)
\end{aligned}
$$

for a properly chosen $\epsilon$ and finite $E\left[\varphi^{2}(x) x^{-1 / 2}\right]$. Finally, this implies that $E_{X_{1}, X_{2}}^{2}\left[H_{T}^{2}\left(X_{1}, X_{2}\right)\right]=O\left(T^{-8} b^{-1}\right)$ and that

$T b^{1 / 4} I-\frac{b^{-1 / 4}}{2 \sqrt{\pi}} E\left[x^{-1 / 2} \varphi(x)\right] \stackrel{d}{\longrightarrow} N\left(0, \frac{1}{\sqrt{\pi}} E\left[\varphi^{2}(x) x^{-1 / 2} f(x)\right]\right)$.

\section{Beta kernel functionals}

I derive the asymptotic behaviour of beta kernel functionals using the same approach as before, that is, I consider the decomposition $I=$ $I_{1}+I_{2}+I_{3}+I_{4}$. The only difference is that $r_{T}(x, X)$ represents now $\varphi^{1 / 2}(x) K_{x / b+1,(1-x) / b+1}\left(X_{t}\right)$. Again, the first term stands for a degenerate U-statistic and contributes with the asymptotic variance, whereas 
the second term provides the asymptotic mean. The third and the fourth terms are, afresh, negligible under proper normalisation provided that the bandwidth $b$ is of order $o\left(T^{-4 / 9}\right)$. Once more, this assumption implies some degree of undersmoothing in view that Chen (1999a) has shown that the optimal bandwidth for beta kernels is of order $O\left(T^{-2 / 5}\right)$.

The limiting distribution of beta kernel functionals is perfectly analogous to that derived for gamma kernels. The only distinction stems from the consideration of the upper bound, which engender a correction inversely proportional to the square root of $x(1-x)$ instead of $x$. More precisely, I show in the sequel that

$$
T b^{1 / 4} I-\frac{b^{-1 / 4}}{2 \sqrt{\pi}} E\left[\frac{\varphi(x)}{\sqrt{x(1-x)}}\right] \stackrel{d}{\longrightarrow} N\left(0, \frac{1}{\sqrt{\pi}} E_{x}\left[\frac{\varphi^{2}(x) f(x)}{\sqrt{x(1-x)}}\right]\right) .
$$

I start by noting that the expectation and variance of a $\mathcal{B}(\mu, v)$ are $v /(\mu+v)$ and $\mu v /\left[(\mu+v)^{2}(\mu+v+1)\right]$, respectively. It is then straightforward to derive the first two moments of $r_{T}(x, X)$. Indeed,

$$
\begin{aligned}
E_{X}\left[r_{T}(x, X)\right] & =\varphi^{1 / 2}(x) \int_{X} K_{x / b+1,(1-x) / b+1}(X) f(X) \mathrm{d} X \\
& =\varphi^{1 / 2}(x) E_{\zeta}[f(\zeta)]
\end{aligned}
$$

where $\zeta \sim \mathcal{B}(x / b+1,(1-x) / b+1)$. Therefore, the mean and variance of $\zeta$ are

$$
\begin{aligned}
& E_{\zeta}=\frac{(1-x) / b+1}{x / b+1+(1-x) / b+1}=\frac{1-x+b}{1+2 b} \\
& V_{\zeta}=\frac{(x / b+1)[(1-x) / b+1]}{(1 / b+2)^{2}(1 / b+3)}=x(1-x) b+O\left(b^{2}\right),
\end{aligned}
$$

respectively. Applying a Taylor expansion yields

$$
\begin{aligned}
E_{\zeta}[f(\zeta)] & =f\left(E_{\zeta}\right)+\frac{1}{2} f^{\prime \prime}(x) V_{\zeta}+o(b) \\
& =f\left(\frac{1-x+b}{1+2 b}\right)+\frac{1}{2} f^{\prime \prime}(x) x(1-x) b+o(b)
\end{aligned}
$$




$$
\begin{aligned}
& =f(x)+f^{\prime}(x) \frac{1-2 x+b-2 b x}{1+2 b}+\frac{1}{2} f^{\prime \prime}(x) x(1-x) b+o(b) \\
& =f(x)+f^{\prime}(x)(1-2 x) \frac{1+b}{1+2 b}+\frac{1}{2} f^{\prime \prime}(x) x(1-x) b+o(b) \\
& =f(x)+\left[f^{\prime}(x)(1-2 x) \frac{1}{2} f^{\prime \prime}(x) x(1-x)\right] b+o(b) \\
& =f(x)+O(b),
\end{aligned}
$$

which implies that the beta kernel estimation of the density function has a uniform bias of order $O(b)$. To sum up,

$$
E_{X}\left[r_{T}(x, X)\right]=\varphi^{1 / 2}(x) f(x)+O(b),
$$

which implies that $\breve{r}_{T}(x, X)=O(b)$.

Now I turn to the second moment of $r_{T}(x, X)$, namely

$$
\begin{aligned}
E_{X}\left[r_{T}^{2}(x, X)\right] & =\varphi(x) \int_{X} K_{x / b+1,(1-x) / b+1}^{2}(X) f(X) \mathrm{d} X \\
& =\varphi(x) A_{b}(x) E_{\eta}[f(\eta)]
\end{aligned}
$$

where

$$
A_{b}(x)=\frac{B[2 x / b+1,2(1-x) / b+1]}{B^{2}[x / b+1,(1-x) / b+1]}
$$

and $\eta \sim \mathcal{B}(2 x / b+1,2(1-x) / b+1)$. The mean and variance of $\eta$ are

$$
\begin{aligned}
& E_{\eta}=\frac{2(1-x) / b+1}{2 x / b+1+2(1-x) / b+1}=\frac{2(1-x)+b}{2(1+b)} \\
& V_{\eta}=\frac{(2 x / b+1)[2(1-x) / b+1]}{(2 / b+2)^{2}(2 / b+3)}=\frac{1}{2} x(1-x) b+O\left(b^{2}\right)
\end{aligned}
$$

respectively, hence applying a Taylor expansion yields

$$
\begin{aligned}
E_{\eta}[f(\eta)] & =f\left(E_{\eta}\right)+\frac{1}{2} f^{\prime \prime}(x) V_{\eta}+o(b) \\
& =f\left(\frac{(2(1-x)+b}{2(1+b)}\right)+\frac{1}{4} f^{\prime \prime}(x) x(1-x) b+o(b)
\end{aligned}
$$




$$
\begin{aligned}
& =f(x)+f^{\prime}(x)(1-2 x) \frac{2+b}{2(1+b)}+\frac{1}{4} f^{\prime \prime}(x) x(1-x) b+o(b) \\
& =f(x)+\frac{1}{2}\left[f^{\prime}(x)(1-2 x)+\frac{1}{2} f^{\prime \prime}(x) x(1-x)\right] b+o(b) \\
& =f(x)+O(b) .
\end{aligned}
$$

Then, it follows that

$$
\begin{aligned}
E\left(I_{2}\right) & =\frac{1}{T} \int_{x} E_{X}\left[r_{T}^{2}(x, X)\right] \mathrm{d} x \\
& =\frac{1}{T} \int_{x} \varphi(x) A_{b}(x)[f(x)+O(b)] \mathrm{d} x \\
& \left.=\frac{1}{T} \int_{x} \varphi(x) A_{b}(x) f(x)\right] \mathrm{d} x+O(1 / T)
\end{aligned}
$$

For $b$ small enough, Chen (1999a) showed that $A_{b}(x)$ may be approximated according to the location of $x$ within the support. More precisely, $x / b$ and $(1-x) / b$ grows without bound as $b$ shrinks to zero in the interior of the support, whereas either $x / b$ or $(1-x) / b$ converges to some non-negative constant $c$ in the boundaries. The approximation is such that

$$
A_{b}(x) \sim \begin{cases}\frac{1}{2 \sqrt{\pi}} b^{-1 / 2}[x(1-x)]^{-1 / 2} & \text { if } x / b \text { and }(1-x) / b \rightarrow \infty \\ \frac{\Gamma(2 c+1) / b}{2^{2 c+1} \Gamma^{2}(c+1)} & \text { if } x / b \text { or }(1-x) / b \rightarrow c,\end{cases}
$$

which implies that $A_{b}(x)$ is of larger order near the boundary. Nonetheless, I show that there is no impact whatsoever in $E\left(I_{2}\right) .^{3}$

Let $\delta=b^{1-\epsilon}$, where $0<\epsilon<1$. Then,

$$
\begin{aligned}
E\left(I_{2}\right) & =\frac{1}{T} \int_{x} \varphi(x) A_{b}(x) f(x) \mathrm{d} x+O(1 / T) \\
& =\frac{1}{T} \int_{0}^{\delta}+\int_{\delta}^{1-\delta}+\int_{1-\delta}^{1} \varphi(x) A_{b}(x) f(x) \mathrm{d} x+O(1 / T)
\end{aligned}
$$

3 This result is analogous to Chen's (1999a) result concerning the variance of the beta kernel estimator. In particular, the variance mounts as $x$ approaches the boundary, but this increase does not affect the integrated variance of the estimator. 


$$
\begin{aligned}
& =\frac{1}{2 \sqrt{\pi} T} \int_{\delta}^{1-\delta} b^{-1 / 2}[x(1-x)]^{-1 / 2} \varphi(x) f(x) \mathrm{d} x+O\left(T^{-1} b^{-\epsilon}\right) \\
& =\frac{b^{-1 / 2}}{2 \sqrt{\pi} T} \int_{0}^{1} \varphi(x)[x(1-x)]^{-1 / 2} f(x) \mathrm{d} x+o\left(T^{-1} b^{-1 / 2}\right)
\end{aligned}
$$

as long as $\epsilon$ is properly chosen and $E[\varphi(x) / \sqrt{x(1-x)}]$ is finite. Therefore, it ensues that

$T b^{1 / 4} E\left(I_{2}\right)=\frac{b^{-1 / 4}}{2 \sqrt{\pi}} E\left[\frac{\varphi(x)}{\sqrt{x(1-x)}}\right]$.

Notice also that

$$
\begin{aligned}
V\left(I_{2}\right) & =\frac{1}{T^{3}} E\left[\int_{x} r_{T}^{2}(x, X) \mathrm{d} x\right]^{2}-\frac{1}{T^{3}} E^{2}\left[\int_{x} r_{T}^{2}(x, X) \mathrm{d} x\right] \\
& =\frac{1}{T^{3}} E\left[\int_{x} r_{T}^{2}(x, X) \mathrm{d} x\right]^{2}-\frac{1}{T^{3}}\left[\int_{x} E r_{T}^{2}(x, X) \mathrm{d} x\right]^{2} \\
& =O\left(T^{-3} b^{-1}\right) .
\end{aligned}
$$

Thus, $V\left(T b^{1 / 4} I_{2}\right)=T^{2} b^{1 / 2} V\left(I_{2}\right)=O\left(T^{-1} b^{-1 / 2}\right)$, which is of order $o(1)$ given the assumption on the bandwidth. Thus, by Chebyshev's inequality,

$$
T b^{1 / 4} I_{2}-\frac{b^{-1 / 4}}{2 \sqrt{\pi}} E\left[\frac{\varphi(x)}{\sqrt{x(1-x)}}\right]=o_{p}(1) .
$$

Applying exactly the same techniques used in the gamma context, it is straightforward to demonstrate that the third and fourth terms are negligible under proper normalisation. Indeed, the fact that the bandwidth is such that $b=o\left(T^{-4 / 9}\right)$ suffices to guarantee that $T b^{1 / 4} I_{3}=o(1)$ and $T b^{1 / 4} I_{4}=o_{p}(1)$. Lastly, it is evident given the previous discussion that $I_{1}=\sum_{s<t} H_{T}\left(X_{t}, X_{s}\right)$, where

$$
H_{T}\left(X_{t}, X_{s}\right)=\frac{2}{T^{2}} \int_{x} \breve{r}_{T}\left(x, X_{t}\right) \breve{r}_{T}\left(x, X_{s}\right) \mathrm{d} x,
$$


is a degenerate U-statistic. Let $V_{H}=\frac{T^{4}}{2} E_{X_{1}, X_{2}}\left[H_{T}^{2}\left(X_{1}, X_{2}\right)\right]$, then

$$
\begin{aligned}
V_{H}= & 2 \int_{X_{1}, X_{2}}\left[\int_{x} \breve{r}_{T}\left(x, X_{1}\right) \breve{r}_{T}\left(x, X_{2}\right) \mathrm{d} x\right]^{2} f\left(X_{1}, X_{2}\right) \mathrm{d}\left(X_{1}, X_{2}\right) \\
= & 2 \int_{x, y}\left[\int_{X} \breve{r}_{T}(x, X) \breve{r}_{T}(y, X) f(X) \mathrm{d} X\right]^{2} \mathrm{~d}(x, y) \\
= & 2 \int_{x, y} \varphi(x) \varphi(y) E_{X}^{2}\left\{\left[K_{x / b+1,(1-x) / b+1}(X)-E_{K(x, b)}\right]\right. \\
& \left.\times\left[K_{y / b+1,(1-y) / b+1}(X)-E_{K(y, b)}\right]\right\} \mathrm{d}(x, y),
\end{aligned}
$$

where $E_{K(u, b)}=E_{X}\left[K_{u / b+1,(1-u) / b+1}(X)\right]$. As before, it turns out that

$V_{H} \simeq 2 \int_{x, y} \varphi(x) \varphi(y)\left[\int_{X} K_{\frac{x}{b}+1, \frac{1-x}{b}+1}(X) K_{\frac{z}{b}+1, \frac{1-y}{b}+1}(X) \mathrm{d} F(X)\right]^{2} \mathrm{~d}(x, y)$

due to the fact that all other terms are of order $O\left(b^{2}\right)$.

Let $g(X)=f(X) K_{x / b+1,(1-x) / b+1}(X)$ and write

$V_{H} \simeq 2 \int_{x, y} \varphi(x) \varphi(y)\left[\int_{X} g(X) \mathrm{d} K_{y / b+1,(1-y) / b+1}(X)\right]^{2} \mathrm{~d}(x, y)$.

It follows from a Taylor expansion that

$$
\begin{aligned}
\int_{X} g(X) & \mathrm{d} K_{y / b+1,(1-y) / b+1}(X) \\
= & E_{\mathcal{B}(y / b+1,(1-y) / b+1)}[g(X)] \\
= & g\left[E_{\mathcal{B}(y / b+1,(1-y) / b+1)}(X)\right]+\frac{1}{2} g^{\prime \prime}(y) V_{\mathcal{B}(y / b+1,(1-y) / b+1)}(X)+o(b) \\
= & g\left(\frac{1-y+b}{1+2 b}\right)+\frac{g^{\prime \prime}(y) y(1-y) b}{2}+o(b) \\
= & g(y)+O(b),
\end{aligned}
$$

which implies that

$$
\begin{aligned}
V_{H} & \simeq 2 \int_{x, y} \varphi(x) \varphi(y)\left[f(y) K_{x / b+1,(1-x) / b+1}(y)\right]^{2} \mathrm{~d}(x, y) \\
& \simeq 2 \int_{x} \varphi(x) \int_{y} \varphi(y) f^{2}(y) K_{x / b+1,(1-x) / b+1}^{2}(y) \mathrm{d} y \mathrm{~d} x \\
& \simeq 2 \int_{x} \varphi(x) \int_{y} h(y) \mathrm{d} K_{x / b+1,(1-x) / b+1}(y) \mathrm{d} x,
\end{aligned}
$$


where $h(y)=\varphi(y) f^{2}(y) K_{x / b+1,(1-x) / b+1}(y)$. Applying another Taylor expansion gives forth that

$$
\begin{aligned}
\int_{y} h(y) \mathrm{d} & K_{x / b+1,(1-x) / b+1}(y) \\
& =E_{\mathcal{B}(x / b+1,(1-x) / b+1)}[h(y)] \\
& =h\left[E_{\mathcal{B}(x / b+1,(1-x) / b+1)}(y)\right]+\frac{1}{2} h^{\prime \prime}(x) V_{\mathcal{B}(x / b+1,(1-x) / b+1)}(y)+o(b) \\
& =h\left(\frac{1-x+b}{1+2 b}\right)+\frac{1}{2} h^{\prime \prime}(x) x(1-x) b+o(b) \\
& =h(x)+O(b) .
\end{aligned}
$$

Therefore,

$$
\begin{aligned}
V_{H} & \simeq 2 \int_{x} \varphi(x) h(x) \mathrm{d} x \\
& \simeq 2 \int_{x} \varphi^{2}(x) f^{2}(x) K_{x / b+1,(1-x) / b+1}(x) \mathrm{d} x \\
& \simeq 2 \int_{x} \varphi^{2}(x)\left[\int_{X} f(X) K_{x / b+1,(1-x) / b+1}^{2}(X) \mathrm{d} X\right] \mathrm{d} F(x) \\
& \simeq 2 \int_{x} \varphi^{2}(x) A_{b}(x)[f(x)+O(b)] \mathrm{d} F(x) \\
& \simeq 2 \int_{x} \varphi^{2}(x) A_{b}(x) f(x) \mathrm{d} F(x) .
\end{aligned}
$$

By decomposing the integral according to $\delta=b^{1-\epsilon}$, it yields

$$
\begin{aligned}
V_{H} & \simeq \int_{0}^{\delta}+\int_{\delta}^{1-\delta}+\int_{1-\delta}^{1} 2 \varphi^{2}(x) A_{b}(x) f(x) \mathrm{d} F(x) \\
& \simeq \frac{b^{-1 / 2}}{\sqrt{\pi}} \int_{\delta}^{1-\delta} \varphi^{2}(x)[x(1-x)]^{-1 / 2} f(x) \mathrm{d} F(x) \\
& \simeq \frac{b^{-1 / 2}}{\sqrt{\pi}} \int_{0}^{1} \varphi^{2}(x)[x(1-x)]^{-1 / 2} f(x) \mathrm{d} F(x)
\end{aligned}
$$

provided that $\epsilon$ is properly chosen and $E\left[\varphi^{2}(x)[x(1-x)]^{-1 / 2}\right]$ is finite. Applying Hall's central limit theorem for degenerate U-statistics completes then the proof. 


\section{References}

Ait-Sahalia, Y. (1996). Testing continuous-time models of the spot interest rate, Review of Financial Studies 9: 385-426.

Aït-Sahalia, Y., Bickel, P. J. and Stoker, T. M. (1998). Goodness-offit tests for regression using kernel methods, Princeton University, University of California at Berkeley, and Massachusetts Institute of Technology.

Bickel, P. J. and Rosenblatt, M. (1973). On some global measures of the deviations of density function estimates, Annals of Statistics 1: 1071-1095.

Chen, S. X. (1999a). Beta kernel estimators for density functions, Computational Statistics and Data Analysis. Forthcoming.

Chen, S. X. (1999b). Probability density function estimation using gamma kernels, School of Statistical Science, La Trobe University.

Fernandes, M. and Grammig, J. (1999). Non-parametric specification tests for conditional duration models, European University Institute and University of Frankfurt.

Hall, P. (1984). Central limit theorem for integrated squared error multivariate nonparametric density estimators, Journal of Multivariate Analysis 14: 1-16.

Müller, H. G. (1984). Smooth optimum kernel estimators of densities, regression curves and modes, Annals of Statistics 12: 766-774. 


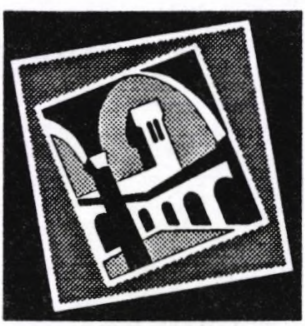

EUI

WORKING

PAPERS

EUI Working Papers are published and distributed by the European University Institute, Florence

Copies can be obtained free of charge

- depending on the availability of stocks - from:

The Publications Officer

European University Institute

Badia Fiesolana

I-50016 San Domenico di Fiesole (FI)

Italy 


\title{
Publications of the European University Institute
}

To

\author{
The Publications Officer \\ European University Institute \\ Badia Fiesolana \\ I-50016 San Domenico di Fiesole (FI) - Italy \\ Telefax No: $+39 / 055 / 4685636$ \\ e-mail: publish@datacomm.iue.it \\ http://www.iue.it
}

From

Name

Address.

$\square$ Please send me a list of EUI Working Papers

$\square$ Please send me a list of EUI book publications

$\square$ Please send me the EUI brochure Academic Year 2000/01

Please send me the following EUI Working Paper(s):

No, Author

Title:

No, Author

Title:

No, Author

Title:

No, Author

Title:

Date

Signature 


\section{Working Papers of the Department of Economics Published since 1999}

ECO No. 99/1

Jian-Ming ZHOU

How to Carry Out Land Consolidation An International Comparison

ECO No. 99/2

Nuala O'DONNELL

Industry Earnings Differentials in Ireland: 1987-1994

ECO No. 99/3

Ray BARRELL/Rebecca RILEY

Equilibrium Unemployment and Labour

Market Flows in the UK

ECO No. 99/4

Klaus ADAM

Learning while Searching for the Best

Alternative

ECO No. 99/5

Guido ASCARI/Juan Angel GARCIA

Relative Wage Concern and the

Keynesian Contract Multiplier

ECO No. 99/6

Guido ASCARI/Juan Angel GARCIA

Price/Wage Staggering and Persistence

ECO No. 99/7

Elena GENNARI

Estimating Money Demand in Italy:

1970-1994

ECO No. 99/8

Marcello D'AMATO/Barbara PISTORESI

Interest Rate Spreads Between Italy and

Germany: 1995-1997

ECO No. 99/9

Søren JOHANSEN

A Small Sample Correction for Tests of

Hypotheses on the Cointegrating Vectors

ECO No. 99/10

Søren JOHANSEN

A Bartlett Correction Factor for Tests on the Cointegrating Relations
ECO No. 99/11

Monika MERZ/Axel

SCHIMMELPFENNIG

Career Choices of German High School

Graduates: Evidence from the German

Socio-Economic Panel

ECO No. 99/12

Fragiskos ARCHONTAKIS

Jordan Matrices on the Equivalence of the I (1) Conditions for VAR Systems

ECO No. 99/13

Étienne BILLETTE de VILLEMEUR

Sequential Decision Processes Make

Behavioural Types Endogenous

ECO No. 99/14

Günther REHME

Public Policies and Education, Economic

Growth and Income Distribution

ECO No. 99/15

Pierpaolo BATTIGALLI/

Marciano SINISCALCHI

Interactive Beliefs and Forward Induction

ECO No. 99/16

Marco FUGAZZA

Search Subsidies vs Hiring Subsidies:

A General Equilibrium Analysis of

Employment Vouchers

ECO No. 99/17

Pierpaolo BATTIGALLI

Rationalizability in Incomplete

Information Games

ECO No. 99/18

Ramon MARIMON/Juan Pablo

NICOLINL/Pedro TELES

Competition and Reputation

ECO No. 99/19

Ramon MARIMON/Fabrizio ZILIBOTTI

Employment and Distributional Effects of

Restricting Working Time 
ECO No. 99/20

Leonor COUTINHO

Euro Exchange Rates: What Can $\mathrm{Be}$

Expected in Terms of Volatility?

ECO No. 99/21

Bernard FINGLETON

Economic Geography with Spatial

Econometrics: A 'Third Way' to Analyse

Economic Development and

'Equilibrium', with Application to the EU

Regions

ECO 99/22

Mike ARTIS/

Massimiliano MARCELLINO

Fiscal Forecasting: The Track Record of the IMF, OECD and EC

\section{ECO 99/23}

Massimo MOTTA/Michele POLO

Leniency Programs and Cartel

Prosecution

\section{ECO 99/24}

Mike ARTIS/Hans-Martin KROLZIG/

Juan TORO

The European Business Cycle

\section{ECO 99/25}

Mathias HOFFMANN

Current Accounts and the Persistence of Global and Country-Specific Shocks: Is Investment Really too Volatile?

\section{ECO 99/26}

Mathias HOFFMANN

National Stochastic Trends and International Macroeconomic Fluctuations: The Role of the Current Account

\section{ECO 99/27}

Gianmarco I.P. OTTAVIANO/

Jacques-François THISSE

Integration, Agglomeration and the

Political Economics of Factor Mobility

\section{ECO 99/28}

Gianmarco I.P. OTTAVIANO

Ad usum delphini: A Primer in 'New

Economic Geography'

\section{ECO 99/29}

Giorgio BASEVI/

Gianmarco I.P. OTTA VIANO

The District Goes Global: Export vs FDI
ECO 99/30

Hans-Martin KROLZIG/Juan TORO

A New Approach to the Analysis of

Shocks and the Cycle in a Model of

Output and Employment

\section{ECO 99/31}

Gianmarco I.P. OTTAVIANO/

Jacques-François THISSE

Monopolistic Competition, Multiproduct

Firms and Optimum Product Diversity

\section{ECO 99/32}

Stefano MANZOCCHI/

Gianmarco I.P. OTTAVIANO

Outsiders in Economic Integration: The

Case of a Transition Economy

ECO 99/33

Roger E.A. FARMER

Two New Keynesian Theories of Sticky

Prices

\section{ECO 99/34}

Rosalind L. BENNETT/

Roger E.A. FARMER

Indeterminacy with Non-Separable Utility

ECO 99/35

Jess BENHABIB/Roger E.A. FARMER

The Monetary Transmission Mechanism

\section{ECO 99/36}

Günther REHME

Distributive Policies and Economic

Growth: An Optimal Taxation Approach

ECO 99/37 - Ray BARRELL/

Karen DURY/Ian HURST

Analysing Monetary and Fiscal Policy

Regimes Using Deterministic and

Stochastic Simulations

ECO 99/38

Chiara FUMAGALLI/Massimo MOTTA

Upstream Mergers, Downstream

Mergers, and Secret Vertical Contracts

\section{ECO 99/39}

Marcel FRATZSCHER

What Causes Currency Crises: Sunspots, Contagion or Fundamentals?

\section{ECO 99/40}

Simone BORGHESI

Intergenerational Altruism and

Sustainable Development 
ECO 99/41

Jeni KLUGMAN/Alexandre KOLEV

The Role of the Safety Net and the

Labour Market on Falling Cash

Consumption in Russia: 1994-96. A

Quintile-Based Decomposition Analysis

ECO 99/42

Günther REHME

Education, Economic Growth and

Personal Income Inequality Across

Countries

ECO 99/43

Günther REHME

Why are the Data at Odds with Theory?

Growth and (Re-)Distributive Policies in

Integrated Economies

ECO 99/44 - Norbert WUTHE

Exchange Rate Volatility: The Impact of Learning Behaviour and the Institutional Framework - A Market Microstructure Approach

\section{ECO 2000/1}

Marcelo FERNANDES

Central Limit Theorem for Asymmetric

Kernel Functionals 


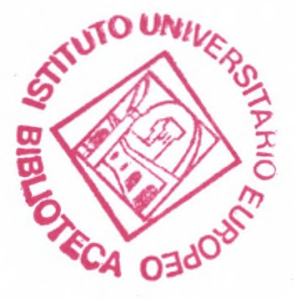

